\title{
Optimal Skip-Stop Schedule under Mixed Traffic Conditions for Minimizing Travel Time of Passengers
}

\author{
Sun Feng, ${ }^{1,2}$ Zhu Wen-tao, ${ }^{3}$ Ye Ying, ${ }^{3}$ and Wang Dian-hai ${ }^{3}$ \\ ${ }^{1}$ College of Transportation, Shandong University of Technology, Zibo 255049, China \\ ${ }^{2}$ College of Transportation, Jilin University, Changchun 130025, China \\ ${ }^{3}$ College of Civil Engineering and Architecture, Zhejiang University, Hangzhou 310058, China
}

Correspondence should be addressed to Wang Dian-hai; wangdianhai@sohu.com

Received 24 July 2013; Revised 15 September 2013; Accepted 4 October 2013

Academic Editor: Wuhong Wang

Copyright (C) 2013 Sun Feng et al. This is an open access article distributed under the Creative Commons Attribution License, which permits unrestricted use, distribution, and reproduction in any medium, provided the original work is properly cited.

\begin{abstract}
Given the lower efficiency resulting from the overload of bus stops, the capacity and travel time of passengers influenced by skipstop operation are analyzed under mixed traffic conditions, and the travel time models of buses and cars are developed, respectively. This paper proposes an optimization model for designing skip-stop service that can minimize the total travel time for passengers. Genetic algorithm is adopted for finding the optimal coordination of the stopping stations of overall bus lines in an urban bus corridor. In this paper, Tian-Mu-Shan Road of Hangzhou City is taken as an example. Results show that the total travel time of all travelers becomes 7.03 percent shorter after the implementation of skip-stop operation. The optimization scheme can improve the operating efficiency of the road examined.
\end{abstract}

\section{Introduction}

In the recent years, with the rapid development of public transport, bus stops face an increasing pressure especially during peak hours, their efficiency decreases continuously, and even serious traffic congestion occurs frequently. The fundamental reason for the above problems is that bus demand exceeds the capacity of bus stops, resulting in some buses waiting in the travel lane until the buses occupying the berth entrance. This not only decreases the level of public transit service but also increases the impact between buses and cars at the location of bus stops on the road without exclusive bus lane. With skip-stop bus services that serve only a subset of stops along certain routes, this problem can be alleviated without technological improvement. For bus passengers, skip-stop services mean improved service levels in the form of lower travel time due to fewer stops and higher between-stop speed. When the skip-stop schedule is adopted by buses, it will reduce the impact of stopping buses on the cars at the location of bus stops, which will increase the car users' travel speed. In actual practice, skip-stop services in systems such as Transmilenio (Bogota, Colombia) and Metro Rapid (Los Angeles, CA, USA) have been proven to be highly effective [1-3]. In China, Shanghai and Shenzhen have also started implementing this operating strategy. However, most cities in China use regular service which is the all-stop operational scheme considering the operational complexity and lack of methodology in modeling an optimal operational scheme.

The literature has a considerable amount of work on the transit operation optimization. Eberlein [4] formulated the stop-skipping problem as an integer nonlinear programming model with both quadratic objective functions and constraints. The real-time scheduling strategy aiming to strike an optimal balance between the benefits of operators and passengers was formulated by $\mathrm{Fu}$ et al. [5]. In this study, the problem is again formulated as a nonlinear 0-1 integer programming problem. Leiva et al. [3] developed an optimization method for designing skip-stop services that minimize the social costs of a segregated bus lane assuming known trip demand. An optimization method is proposed for designing skip-stop services which minimize both the travel time for passengers and the operating cost of an urban bus corridor by optimizing the transport services with different arriving frequencies of various types of buses [6]. In addition, Ceder and Wilson [7], Fan and Machemehl [8], and 
Mauttone and Urquhart [9] determined a set of routes and their respective frequencies by minimizing the sum of users and transit operator costs in a given network and trip demand O-D matrix.

In those studies, the optimization methods are developed inside the transit system, while ignoring the interaction of private and transit vehicle flows. However, there are some roads without exclusive bus lanes in the cities of China. Ignoring this will lead to inaccurate estimates of travel time. Moreover, most of the previous studies were done only for a congested bus line, and they lack the analysis of the overall bus lines that passed by the stops. With these arguments as motivation, this study proposes an optimization method for designing skip-stop services that can minimize the total travel time for passengers under mixed traffic conditions by analyzing the mutual influence between buses and cars, and it uses genetic algorithm to find the optimal coordination of the stop stations of bus lines based on the objective function.

The following notations are used in describing the models in this paper:

$C_{l}$ is the capacity of link;

$C_{0}$ is the basic traffic capacity;

$f_{s}$ is the adjustment factor for bus stops;

$T_{e}$ is the impacting time by bus stops, which is determined by the number of stopping buses at this stop;

$p$ is the probability of no bus being serviced;

$\tau$ is the average dwell time of buses;

$\lambda$ is the average bus arrival rate;

$\lambda_{1}$ is the average arrival rate of buses that stopped at this stop.

$S$ is the number of loading areas;

$N$ is the number of lanes in one direction, $N \geq 2$;

$T(q)$ is the average travel time of vehicle on link $a$;

$T_{0}$ is the free-flow travel time on link $a$;

$q$ is the volume of traffic on link $i$;

$\alpha, \beta$ are parameters;

$i$ is the index of bus line, $i=0,1, \ldots, m$;

$I$ is the set of all bus lines in the network;

$A$ is the set of all links in the network;

$J$ is the set of all stations stopped and skipped by bus line $i$;

$q_{a}^{b, c}$ is the bus volume or car volume on link $a$;

$t_{a}^{c}$ is the in-vehicle travel time on link $a$ by mode car;

$t_{i, k}^{b}$ is the in-vehicle travel time on link $k$ by mode bus;

$n_{i, k}^{b}$ is the average number of passengers on bus line $i$ on link $k$;

$n_{a}^{c}$ is the average number of passengers on car;

$W_{j}$ is the total waiting time for passengers at stop $j$, which includes alighting and transferring at stop $j$;

$K_{i}$ is the set of all links where bus line $i$ runs;
TABLE 1: Capacity of on-line linear stops (buses/h).

\begin{tabular}{lccc}
\hline \multirow{2}{*}{ Number of loading areas } & \multicolumn{3}{c}{ Dwell time (s) } \\
& 15 & 30 & 45 \\
\hline 1 & 63 & 43 & 32 \\
2 & 117 & 80 & 59 \\
3 & 154 & 105 & 78 \\
\hline
\end{tabular}

Assuming $15 \mathrm{~s}$ clearance time, 25 percent queue probability, 60 percent coefficient of variation of dwell times, and $0.5 \mathrm{~g} / \mathrm{C}$.

$Q_{i, j}$ is the number of passengers on buses of line $i$ at stop $j$;

$\tau_{i, j}$ is the dwell time of buses of line $i$ at stop $j$;

$U_{i, j}$ is the number of passengers boarding buses of line $i$ at stop $j$;

$V_{i, j}$ is the number of passengers alighting buses of line $i$ at stop $j$;

$w_{i, j}$ is the waiting time of passengers boarding buses of line $i$ at stop $j$;

$y_{i j}$ is the decision variables to indicate stop status of bus line $i$ at stop $j$, which is equal to 1 if bus line $i$ stops at station $j$ and 0 otherwise;

$L_{j}$ is the distance between station $j$ and $j+1$;

$f_{i}$ is the schedule frequency of bus line $i$;

$t_{\mathrm{oc}}$ is the time of door opening and closing (s);

$a$ is passenger boarding time (s/p);

$b$ is passenger alighting time $(\mathrm{s} / \mathrm{p})$;

$\theta$ is the transfer penalty;

$x_{j}$ is the number of buses stopping at stop $j$ with skipstop operation;

$B_{j}$ is the capacity of stop $j$, from Table $1[1]$.

\section{Travel Time Model under Mixed Traffic Conditions}

The travel time depends on the traffic volume and capacity of links, which is influenced by the number of stopping buses at a stop station under mixed traffic conditions. The BPR (Bureau of Public Roads) function, the most classic model, describes the link performance, which states the relationship between resistance and traffic volume [10]. In this section, the impact of bus stops on the link capacity is analyzed, and then the model of computing travel time is established based on the data of simulation runs.

2.1. Link Capacity. Curbside bus stops interfere with traffic flows, as the buses stop in the travel lane, resulting in a "bottleneck" (the reduction in the road width) at the location of the stops. Bus bays interfere with passing vehicles primarily, while buses maneuver to pull into and out of 
the stops. In general, the capacity is calculated by adding adjustment factor for bus stops [1], as follows:

$$
C_{l}=C_{0} \cdot\left(1-f_{s}\right)=C_{0} \cdot\left(1-\frac{T_{e}}{3600}\right)
$$

When all passing buses stop at this stop, the impacting time is determined by the numbers of loading areas and passengers getting on and off buses. If the bus stop is considered as a queuing system, the probability of no bus being serviced can be calculated by the following equation [11]:

$$
p=\left[\sum_{k=0}^{S-1} \frac{(\lambda \tau)^{k}}{k !}+\frac{(\lambda \tau)^{S}}{S !(1-\lambda \tau / S)}\right]^{-1}
$$

So the impacting time by bus stops is calculated by

$$
T_{e}=3600 \cdot(1-p)
$$

When skip-stop service is adopted, there are two possible scenarios at stops in a skip-stop operation (1) There is no bus being serviced when skip-stopped bus $N$ arrives at this stop. (2) There is one or more buses being serviced when skipstopped bus $N$ arrives at this stop. In the first scenario, the bus passes directly through with no impact on the capacity of the adjacent lane. In the second scenario, the bus would pass buses being serviced on the curb lane. The lane changing behavior will affect the operation of the vehicle in the adjacent lane and result in the dropping of capacity. According to HCM2000, the affecting time is 4 seconds when one bus uses the adjacent lane.

The probability of the first scenario is determined by the number of buses stopped at this stop, which is computed by the following equation:

$$
p_{1}=\left[\sum_{k=0}^{S-1} \frac{\left(\lambda_{1} \tau\right)^{k}}{k !}+\frac{\left(\lambda_{1} \tau\right)^{S}}{S !\left(1-\lambda_{1} \tau / S\right)}\right]^{-1}
$$

The impacting time in a skip-stop operation is approximately calculated using

$$
\begin{aligned}
T_{e}= & 3600\left(1-p_{1}\right)+4 \times 3600\left(\lambda-\lambda_{1}\right)\left(1-p_{1}\right) \\
= & 3600\left(1+4\left(\lambda-\lambda_{1}\right)\right) \\
& \times\left(1-\left[\sum_{k=0}^{S-1} \frac{\left(\lambda_{1} \tau\right)^{k}}{k !}+\frac{\left(\lambda_{1} \tau\right)^{S}}{S !\left(1-\left(\lambda_{1} \tau\right) / S\right)}\right]^{-1}\right) .
\end{aligned}
$$

TABLE 2: Comparison of the investigated values and simulation results.

\begin{tabular}{lcc}
\hline Statistical parameters & Investigated data & Simulated data \\
\hline Sample size & 390 & 683 \\
Mean of speed & 22.3 & 23.1 \\
Variance of speed & 21.3 & 34.3 \\
\hline
\end{tabular}

Therefore, the capacity of links influenced by bus stops is calculated by

$$
\begin{aligned}
C_{l} & =C_{0} \cdot\left(N-\frac{T_{e}}{3600}\right) \\
& =C_{0}\left(N-\left(1+4\left(\lambda-\lambda_{1}\right)\right)\right.
\end{aligned}
$$

$$
\left.\times\left(1-\left[\sum_{k=0}^{S-1} \frac{\left(\lambda_{1} \tau\right)^{k}}{k !}+\frac{\left(\lambda_{1} \tau\right)^{S}}{S !\left(1-\left(\lambda_{1} \tau\right) / S\right)}\right]^{-1}\right)\right)
$$

2.2. Simulation Model Development and Validation. The classic BPR function is calibrated using the traffic data of highway. Therefore, the parameters need to be recalibrated when BPR function is used to describe the performance of urban roads. It is difficult to survey traffic operating data under various conditions. So the parameters of BPR function are calibrated using the simulated data in this paper.

Based on the paper's objectives as well as the required details in the analysis, VISSIM (version 4.2) is employed in this paper. The simulation model is developed using VISSIM based on the surveyed data. For the purpose of model validation, the mean speed of values cars and buses observed on the field and simulated by VISSIM are compared. The comparison results of different types of vehicles are shown in Table 2 .

A paired $t$-test of null hypothesis of no-mean difference is performed to check for the match between simulated and observed average speed of values vehicles. The calculated value of $t$ is 0.6095 against the critical values (from " $t$ " table) of 1.648. It is found that the observed and simulated average speed values agree at 5\% level of significance $(95 \%$ confidence limit). It can be seen that the simulated speed values significantly replicate the observed field speed for all vehicle types, and the VISSIM model can be applied to study the characteristics of traffic flow on urban road links.

2.3. Calibration of Travel Time Model. The BPR function is expressed as follows [10]:

$$
T(q)=T_{0}\left[1+\alpha\left(\frac{q}{C_{l}}\right)^{\beta}\right] .
$$




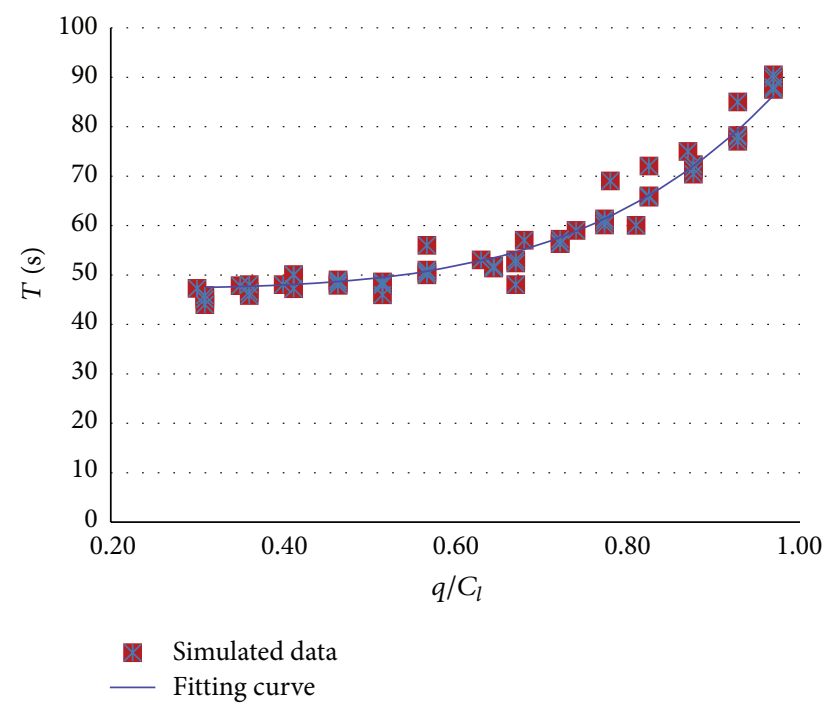

FIGURE 1: Relationship between the travel time of car and $q / C_{l}$ on the segment.

In order to improve the reliability of VISSIM model, the simulation runs are made with random number seeds ranging from 41 to 45 , and the average of the five values is taken as the final model output. The travel time outputted from the model is as shown in Figure 1.

In this paper, BPR model is calibrated by the method of Least-squares. First, BPR model should be deformed as a linear function by logarithmic transformation [12].

$$
\ln \left(\frac{V_{0}}{V}-1\right)=\ln \alpha+\beta \ln \left(\frac{q}{C_{l}}\right) .
$$

Denoting $\ln (q / c)$ by $X, \ln \left(V_{0} / V-1\right)$ by $Y, \beta$ by $A$, and $\ln \alpha$ by $B$, then (8) is transformed to

$$
Y=A X+B
$$

Second, these above data are transformed to $X$ and $Y$. Third, the values of $A$ and $B$ are estimated by the method of least-squares; then, the values $\alpha$ and $\beta$ are 4.2 and 0.87 , respectively. Therefore, the travel time of cars is obtained when there is an on-line curbside bus stop on the road, as shown in the following:

$$
T(q)=T_{0}\left[1+0.87\left(\frac{q}{C_{l}}\right)^{4.2}\right]
$$

\section{Skip-Stop Design Model Formulation}

As indicated in the previous studies, the skip-stop problem can be formulated as a nonlinear 0-1 integer programming problem, with the binary integer variables representing which stops to be skipped by the control vehicles. In this study, for the purpose of solving the exact problem, the stop-skipping problem will be formulated again as a nonlinear 0-1 integer programming problem.

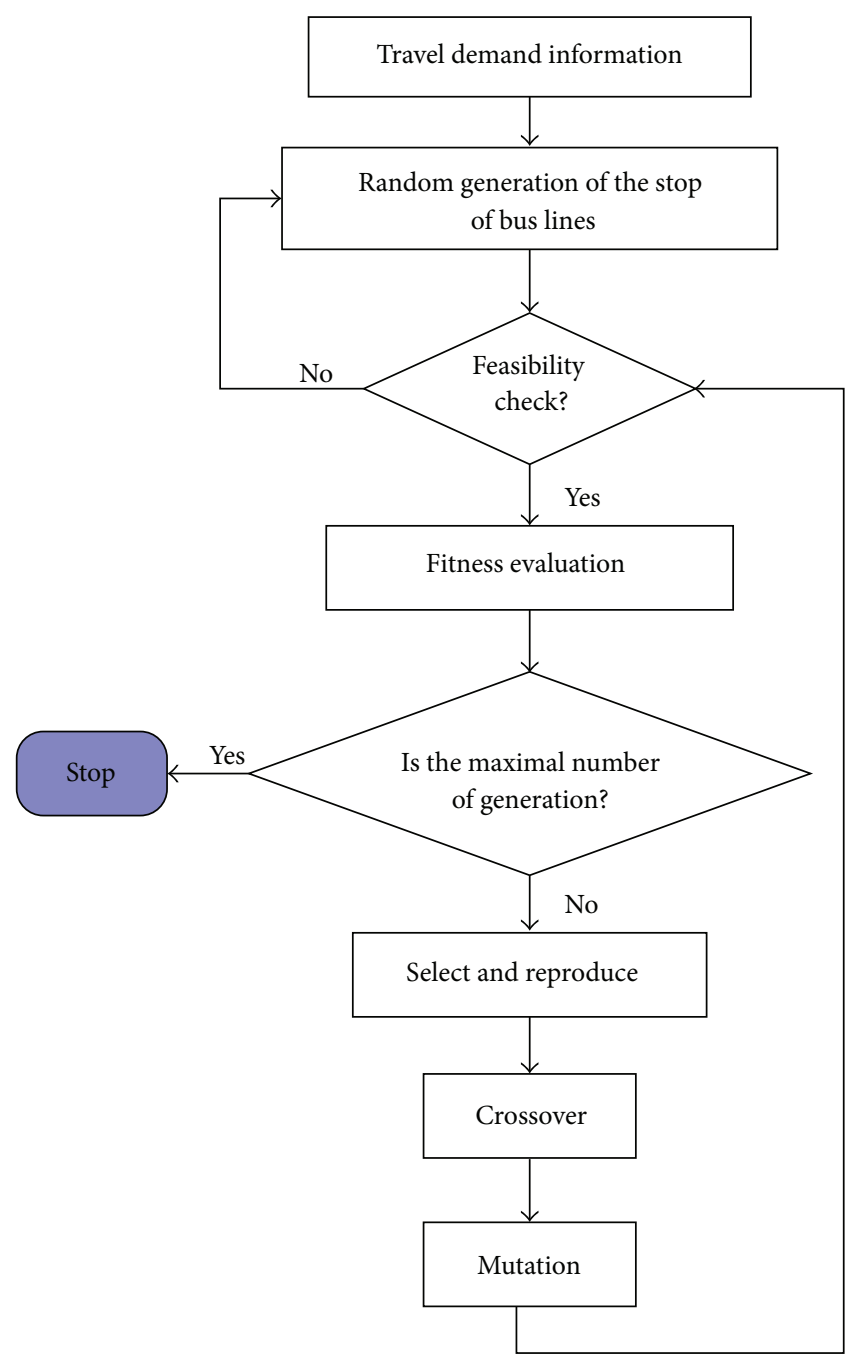

FIGURE 2: Overall procedure for finding an optimal scenario.

3.1. Assumptions. In order to analyze the process, the following assumptions are made in this study.

(1) The total travel demand between any origin and destination node pair (O-D matrix) is fixed and remains the same during the analysis period. It is assumed that the travel demand is not affected by the introduction of skip-stop operation.

(2) There are only two modes of traffic (buses and cars) utilizing the network.

(3) Dwell time at each stop is determined by the number of passengers getting on and off the bus.

(4) Passengers are uniformly distributed throughout the area.

(5) A passenger would not leave the platform, and she or he would not wait more than two times.

3.2. Skip-Stop Design Models under Mixed Traffic Conditions. Run time models are usually used in understanding the existing service and evaluating several transit planning and 
Table 3: Traffic data on Tian-Mu-Shan Road.

\begin{tabular}{lccc}
\hline No. & Bus stop & Car volume (veh/h) & Distance between adjacent stops $(\mathrm{m})$ \\
\hline 1 & Gucui Intersection & 930 & - \\
2 & Xueyuan Intersection & 975 & 280 \\
3 & Qingfeng Village, West & 893 & 522 \\
4 & Qingfeng Village, East & 920 & 395 \\
5 & Xixi District & 823 & 345 \\
6 & Bazi Bridge & 760 & 550 \\
7 & Macheng Intersection & 726 & 270 \\
8 & City Government & 620 & 510 \\
9 & Hushu Intersection & 723 & 280 \\
10 & Hangzhou Building & 770 & 420 \\
11 & Zhongshan Intersection & 780 & 410 \\
\hline
\end{tabular}

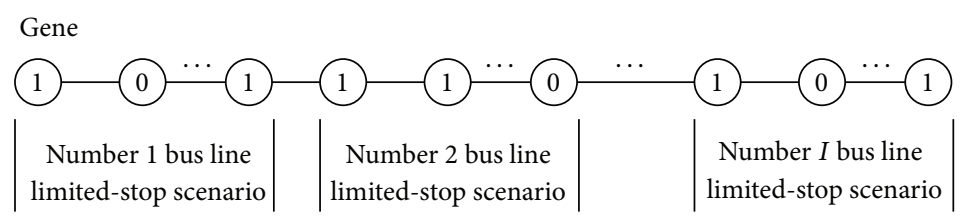

FIGURE 3: The coding illustration of a chromosome for the genetic algorithm.

operation strategies $[13,14]$. So the objective function is denoted as follows:

$$
\begin{aligned}
\operatorname{Min} Z= & \left(\sum_{i \in I} \sum_{k \in K_{i}} n_{i, k}^{b} t_{i, k}^{b}\left(x_{j}\right)+\sum_{j \in J} W_{j}\right) \\
& +\sum_{a \in A} q_{a}^{c} \times n_{a}^{c} \times t_{a}^{c}\left(x_{j}\right)
\end{aligned}
$$

s.t.

$$
\begin{gathered}
W_{j}=\sum_{i \in I} y_{i, j} \times\left(U_{i, j} \times w_{i, j}+Q_{i, j} \times \tau_{i, j}\right) \\
+\theta \times\left(1-y_{i, j}\right) \times\left(U_{i, j}+V_{i, j}\right), \quad \forall j \in J, \\
w_{i, j}=\theta=\frac{1}{\left(2 \cdot f_{i}\right)} \quad \forall i=0,1, \ldots, n, \forall j \in J, \\
\tau_{i, j}=\operatorname{Max}\left\{a \cdot U_{i, j}, b \cdot V_{i, j}\right\}+t_{\mathrm{oc}}, \quad \forall i=0,1, \ldots, n, \forall j \in J, \\
x_{j}=\sum_{i \in I} y_{i, j} \cdot f_{i}, \quad \forall i=0,1, \ldots, n, \forall j \in J, \\
Q_{i, j+1}=Q_{i, j}+U_{i, j}-V_{i, j+1}, \quad \forall i=0,1, \ldots, n, \forall j \in J, \\
y_{i, j} \in\{0,1\}, \quad \forall i=0,1, \ldots, n, \forall j \in J, \\
y_{i, j}+y_{i, j+1} \geq 1, \quad \forall i=0,1, \ldots, n, \forall j \in J, \\
x_{j} \leq B_{j}, \quad \forall j \in J .
\end{gathered}
$$

The first term in the objective function is the total travel time by buses, which includes in-vehicle travel time and total waiting time. The next term represents the total travel time by cars. Equation (12) includes two components. The first component is the sum of waiting time of boarding and invehicle passengers when buses of line $i$ make a stop $j$, while the second component is the transfer time of passengers with either their origin or destination stop $j$ being skipped. Equation (13) indicates that the average waiting time and the transfer time of passengers boarding buses of line $i$ at the stop $j$ are equal to half the headway of bus line $i$ assuming random arrival. Equation (14) estimates the bus dwell time at each stop based on the number of passengers who will board and alight at the stop, denoted by $U_{i j}$ and $V_{i j}$, respectively. Equation (15) shows that the number of buses stopping at the stop $j$ depends on the schedule frequency of bus line $i$ and whether the bus line $i$ will make stop $j$. Equation (16) states that the number of passengers on buses of line $i$ at stop $j+1$ is equal to the number of passengers on buses of line $i$ at stop $j$ plus the number of passengers boarding buses of line $i$ at stop $j$ minus the number of passengers alighting buses of line $i$ at stop $j+1$. The decision variable is $y_{i j}$ by which the system managers try to minimize their objective function $(Z)$. Equation (18) specifies that two adjacent stops are not both skipped by any bus line. Equation (19) ensures that the number of buses stopping at stop $j$ cannot exceed its capacity.

\section{Optimization Process Using Genetic Algorithm}

The proposed model is a nonlinear programming problem which associates the zero-one variables, and the parameters are tightly related to each other. It is hardly solved with conventional solution methods. The genetic algorithm (GA) is a heuristic search method that imitates the process of natural evolution [15]. It is motivated by the principles of 


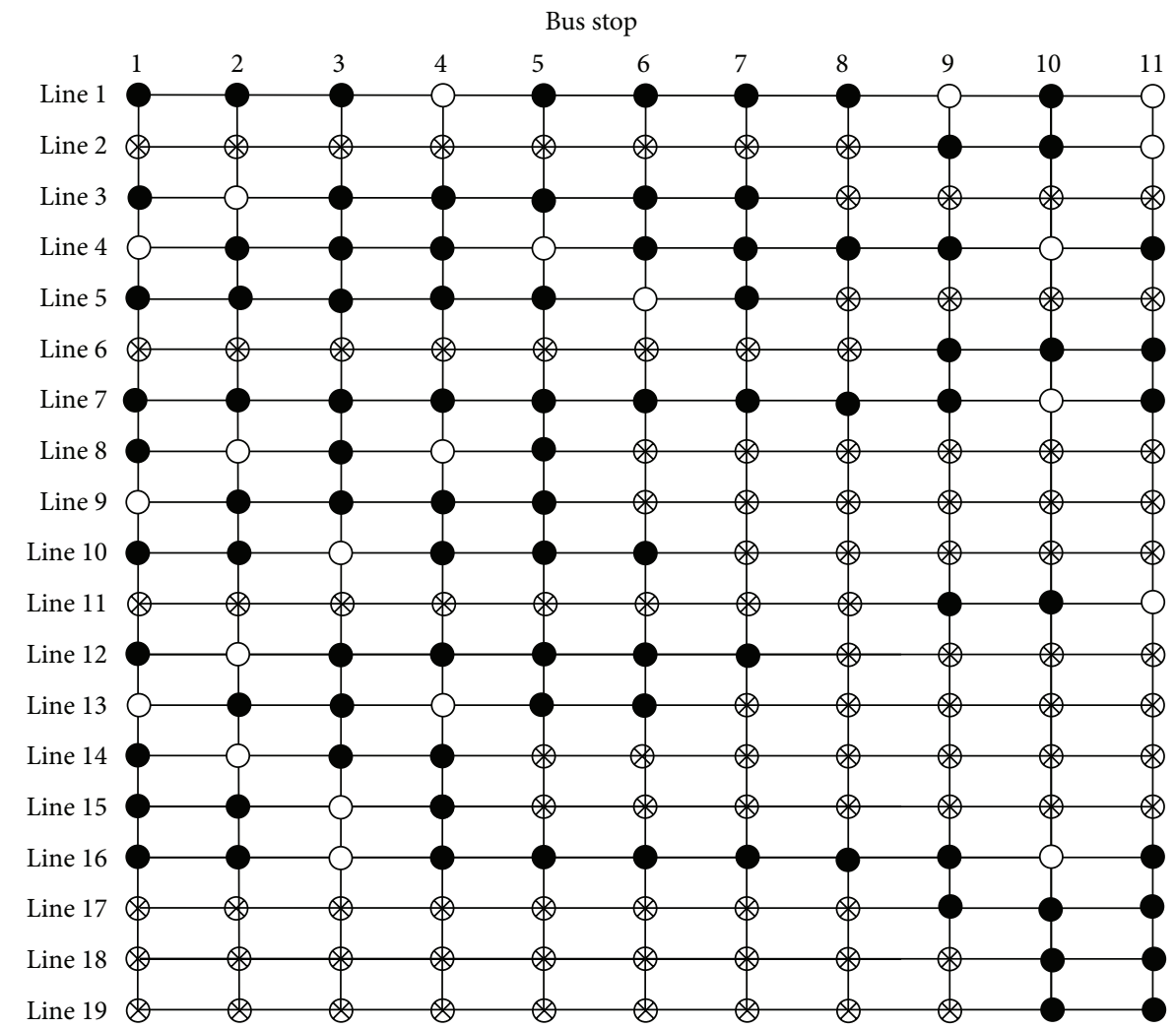

FIGURE 4: The diagram of skip-stop operation scenario. Note that “•” represents stopping, “”” represents skipping, and “ $\otimes$ ” represents not passing by.

TABLE 4: Numbers of passengers boarding and alighting at stops (from west to east).

\begin{tabular}{|c|c|c|c|c|c|c|c|c|c|c|c|}
\hline \multirow{2}{*}{ Line } & \multicolumn{11}{|c|}{ Stop } \\
\hline & 1 & 2 & 3 & 4 & 5 & 6 & 7 & 8 & 9 & 10 & 11 \\
\hline 1 & $19 / 14$ & $14 / 45$ & $24 / 41$ & $27 / 63$ & $42 / 56$ & $36 / 33$ & $16 / 22$ & $49 / 65$ & $21 / 34$ & $28 / 58$ & $16 / 22$ \\
\hline 2 & - & - & - & - & - & - & - & - & $18 / 57$ & $18 / 49$ & $21 / 34$ \\
\hline 3 & $26 / 18$ & $6 / 23$ & $14 / 26$ & $44 / 58$ & $40 / 48$ & $26 / 57$ & $44 / 36$ & - & - & - & - \\
\hline 4 & $13 / 8$ & $22 / 18$ & $28 / 33$ & $26 / 63$ & $28 / 23$ & $14 / 41$ & $20 / 34$ & $54 / 38$ & $24 / 33$ & $17 / 55$ & $19 / 27$ \\
\hline 5 & $18 / 20$ & $10 / 28$ & $8 / 28$ & $25 / 87$ & $26 / 34$ & $37 / 38$ & $32 / 43$ & - & - & - & - \\
\hline 6 & - & - & - & - & - & - & - & - & $17 / 51$ & $20 / 77$ & $36 / 45$ \\
\hline 7 & $17 / 37$ & $8 / 32$ & $6 / 46$ & $14 / 60$ & $34 / 50$ & $23 / 45$ & $10 / 22$ & $58 / 68$ & $31 / 36$ & $7 / 40$ & $24 / 36$ \\
\hline 8 & $22 / 12$ & $12 / 16$ & $3 / 34$ & $26 / 47$ & $29 / 64$ & - & - & - & - & - & - \\
\hline 9 & $29 / 13$ & $6 / 22$ & $5 / 52$ & $17 / 56$ & $38 / 35$ & - & - & - & - & - & - \\
\hline 10 & $12 / 21$ & $12 / 34$ & $26 / 26$ & $40 / 51$ & $22 / 52$ & $49 / 44$ & - & - & - & - & - \\
\hline 11 & - & - & - & - & - & - & - & - & $29 / 39$ & $16 / 62$ & $35 / 24$ \\
\hline 12 & $21 / 22$ & $13 / 25$ & $21 / 76$ & $40 / 62$ & $57 / 38$ & $27 / 67$ & $68 / 47$ & - & - & - & - \\
\hline 13 & $34 / 14$ & $8 / 27$ & $8 / 38$ & $32 / 40$ & $18 / 41$ & $50 / 76$ & - & - & - & - & - \\
\hline 14 & $17 / 18$ & $10 / 16$ & $12 / 14$ & $14 / 58$ & - & - & - & - & - & - & - \\
\hline 15 & $24 / 26$ & $8 / 28$ & $6 / 36$ & $11 / 44$ & - & - & - & - & - & - & - \\
\hline 16 & $16 / 19$ & $6 / 46$ & $19 / 40$ & $44 / 61$ & $64 / 69$ & $44 / 57$ & $30 / 26$ & $34 / 56$ & $10 / 28$ & $25 / 57$ & $12 / 34$ \\
\hline 17 & - & - & - & - & - & - & - & - & $13 / 55$ & $10 / 65$ & $35 / 62$ \\
\hline 18 & - & - & - & - & - & - & - & - & - & $52 / 82$ & $46 / 40$ \\
\hline 19 & - & - & - & - & - & - & - & - & - & $37 / 75$ & $36 / 66$ \\
\hline
\end{tabular}


TABLE 5: The optimal scenario of skip-stop operation.

\begin{tabular}{|c|c|c|c|c|c|c|c|c|c|c|c|}
\hline \multirow{2}{*}{ Line } & \multicolumn{11}{|c|}{ Stop } \\
\hline & 1 & 2 & 3 & 4 & 5 & 6 & 7 & 8 & 9 & 10 & 11 \\
\hline 1 & 1 & 1 & 1 & 0 & 1 & 1 & 1 & 1 & 0 & 1 & 0 \\
\hline 2 & - & - & - & - & - & - & - & - & 1 & 1 & 0 \\
\hline 3 & 1 & 0 & 1 & 1 & 1 & 1 & 1 & - & - & - & - \\
\hline 4 & 0 & 1 & 1 & 1 & 0 & 1 & 1 & 1 & 1 & 0 & 1 \\
\hline 5 & 1 & 1 & 1 & 1 & 1 & 0 & 1 & - & - & - & - \\
\hline 6 & - & - & - & - & - & - & - & - & 1 & 1 & 1 \\
\hline 7 & 1 & 1 & 1 & 1 & 1 & 1 & 1 & 1 & 1 & 0 & 1 \\
\hline 8 & 1 & 0 & 1 & 0 & 1 & - & - & - & - & - & - \\
\hline 9 & 0 & 1 & 1 & 1 & 1 & - & - & - & - & - & - \\
\hline 10 & 1 & 1 & 0 & 1 & 1 & 1 & - & - & - & - & - \\
\hline 11 & - & - & - & - & - & - & - & - & 1 & 1 & 0 \\
\hline 12 & 1 & 0 & 1 & 1 & 1 & 1 & 1 & - & - & - & - \\
\hline 13 & 0 & 1 & 1 & 0 & 1 & 1 & - & - & - & - & - \\
\hline 14 & 1 & 0 & 1 & 1 & - & - & - & - & - & - & - \\
\hline 15 & 1 & 1 & 0 & 1 & - & - & - & - & - & - & - \\
\hline 16 & 1 & 1 & 0 & 1 & 1 & 1 & 1 & 1 & 1 & 0 & 1 \\
\hline 17 & - & - & - & - & - & - & - & - & 1 & 1 & 1 \\
\hline 18 & - & - & - & - & - & - & - & - & - & 1 & 1 \\
\hline 19 & - & - & - & - & - & - & - & - & - & 1 & 1 \\
\hline
\end{tabular}

Note that "1" represents stopping, "0" represents skipping, and "_" represents not passing by.

TABLE 6: The comparison between all-stop and skip-stop operations.

\begin{tabular}{lccccc}
\hline Scenario & $\begin{array}{c}\text { Car travelers } \\
\text { Travel time (min) }\end{array}$ & $\begin{array}{c}\text { Bus travelers } \\
\text { Out-off-vehicle time (min) }\end{array}$ & \multicolumn{2}{c}{ Total travel time (min) } \\
\hline Original scenario & 34,966 & 8,505 & 296,553 & 305,058 & 340,024 \\
Optimal scenario & 30,471 & 12,228 & 273,427 & 285,655 & 316,126 \\
Change & $-12.86 \%$ & $43.77 \%$ & $-7.80 \%$ & $-6.36 \%$ & $-7.03 \%$ \\
\hline
\end{tabular}

natural selection and survival of the fittest individuals. This method is commonly used to generate useful solutions to optimization problems [16]. Therefore, the genetic algorithm is used to solve the bilevel programming model and find the optimal coordination of the stopping stations in order to minimize inconveniences for passengers. Based on the above analysis, the procedure for finding an optimal scenario is presented, as shown in Figure 2.

For the genetic algorithm, each gene location in a chromosome represents a possible skip-stop choice, while the vehicles of bus line traverse every station shown in Figure 3, where "1" indicates stops and "0" indicates skips at the corresponding station. In Figure 3, a chromosome is divided into $I$ independent parts. Each part indicates the corresponding operation choices for every bus line.

\section{Case Study}

5.1. Traffic Survey. This paper takes Tian-Mu-Shan Road (from Wan-Tang Road to Zhong-Shan Road) as an example, which is a heavy-demand corridor of Hangzhou City. The examined period is the morning peak. The traffic volume and the number of passengers getting on and off at 11 stops are collected, as shown in Tables 3 and 4 .

5.2. Results. Parameters values of the model and the algorithm are shown as follows: the population of chromosomes (individuals) for each generation $N=50$, the crossover probability $P_{c}=0.5$, the mutation probability $P_{m}=0.01$, the biggest generation GenMax $=200, a=b=1 \mathrm{~m} / \mathrm{s}^{2}$, and $L=500 \mathrm{~m}$. The optimization process is programmed using MATLAB software. Then, these data of Tables 3 and 4 are input to program, and an optimal scenario is outputted, as shown in Figure 4 and Table 5.

The travel times of all travelers before and after the optimization are compared, as shown in Table 6. It can be seen that the travel time of car travelers becomes 12.86 percent shorter than that with original all-stop operation, and the travel time of bus travelers reduces 6.36 percent due to skipping stations at the same time. The total travel time of all travelers becomes 7.03 percent shorter after the implementation of skip-stop operation. Results show that the optimization scheme can improve the overall operating efficiency of road network. 


\section{Conclusions}

The flow capacity is adjusted at the section of stop zone based on the analysis of the mutual influence between buses and cars at bus stops. BPR function including the modified capacity is calibrated using traffic simulation. Then, this paper proposes an optimization model for designing skip-stop service that can minimize the total travel time of passengers under mixed traffic conditions. The genetic algorithm is used to find the optimal coordination of the stopping stations of overall bus lines in an urban bus corridor. The validation of the model and the algorithm have been proved with the help of a real-world case. Results show that the operational efficiency of buses and cars is improved with skip-stop operation under mixed traffic conditions. Although skip-stop operation is complicated for the operators and it can confuse passengers at the beginning of the service, it can certainly reduce passengers' total travel time. It should be noted that the dwell time of a bus is assumed to be based on the number of passengers boarding and alighting, as a matter of fact, which is also influenced by the number of stopping buses at the stop. Therefore, an interesting project for future research would be to propose an optimization method for designing skip-stop services with the consideration of the bus operating features at stops.

\section{Conflict of Interests}

The authors declare that there is no conflict of interests regarding the publication of this paper.

\section{Acknowledgment}

This work is supported by the National Natural Science Foundation of China (no. 51278454).

\section{References}

[1] Transportation Research Board, Highway Capacity Manual, National Research Council, Washington, DC, USA, 2000.

[2] Transportation Research Board, TCRP Report 100: Transit Capacity and Quality of Service Manual, National Research Council, Washington, DC, USA, 2nd edition, 2003.

[3] C. Leiva, J. C. Muñoz, R. Giesen, and H. Larrain, "Design of limited-stop services for an urban bus corridor with capacity constraints," Transportation Research B, vol. 44, no. 10, pp. 11861201, 2010.

[4] X. J. Eberlein, Real-time control strategies in transit operations: models and analysis [Ph.D. dissertation], Department of Civil and Environmental Engineering, Massachusetts Institute of Technology, Boston, Mass, USA, 1995.

[5] L. Fu, Q. Liu, and P. Calamai, "Real-time optimization model for dynamic scheduling of transit operations," Transportation Research Record, no. 1857, pp. 48-55, 2003.

[6] H. Niu, "Determination of the skip-stop scheduling for a congested transit line by bi-level genetic algorithm," International Journal of Computational Intelligence Systems, vol. 6, no. 4, pp. 1158-1167, 2011.

[7] A. Ceder and N. H. M. Wilson, "Bus network design," Transportation Research B, vol. 20, no. 4, pp. 331-344, 1986.
[8] W. Fan and R. B. Machemehl, "Using a simulated annealing algorithm to solve the transit route network design problem," Journal of Transportation Engineering, vol. 132, no. 2, pp. 122132, 2006.

[9] A. Mauttone and M. E. Urquhart, "A route set construction algorithm for the transit network design problem," Computers and Operations Research, vol. 36, no. 8, pp. 2440-2449, 2009.

[10] Y. Sheffi, Urban Transportation Networks: Equilibrium Analysis with Mathematical Programming Methods, Prentice-Hall, Englewood Cliffs, NJ, USA, 1985, (American).

[11] D. Gross, J. F. Shortle, J. M. Thompson, and C. M. Harris, Fundamentals of Queueing Theory, John Wiley \& Sons, Hoboken, NJ, USA, 2008, (American).

[12] M. Dongfang, W. Dianhai, B. Yiming, and S. Di, "A method of signal timing optimization for spillover dissipation in urban street networks," Mathematical Problems in Engineering, vol. 2013, Article ID 580546, 9 pages, 2013.

[13] M. Mesbah, M. Sarvi, and G. Currie, "New methodology for optimizing transit priority at the network level," Transportation Research Record, no. 2089, pp. 93-100, 2008.

[14] L. Y. Yao, L. S. Sun, W. H. Wang, and H. Xiong, "Adaptability analysis of service facilities in transfer subway stations," Mathematical Problems in Engineering, vol. 2012, Article ID 701852, 12 pages, 2012.

[15] G. Lin, P. Liang, P. Schonfeld, and R. Larson, "Adaptive control of transit operations," Final Report for Project MD-26-7002, University of Maryland, College Park, Md, USA, 1995.

[16] Y. Yin, "Genetic-algorithms-based approach for bilevel programming models," Journal of Transportation Engineering, vol. 126, no. 2, pp. 115-119, 2000. 


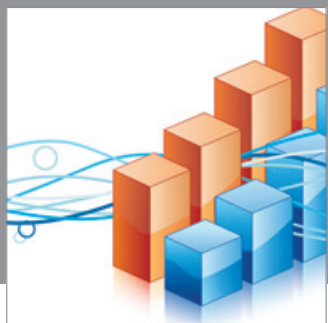

Advances in

Operations Research

mansans

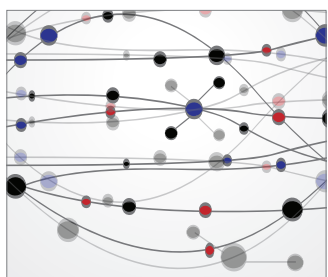

The Scientific World Journal
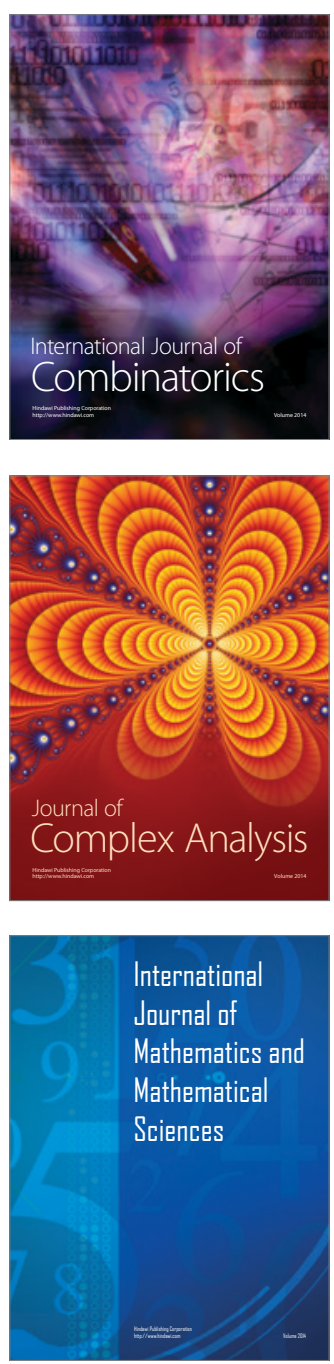
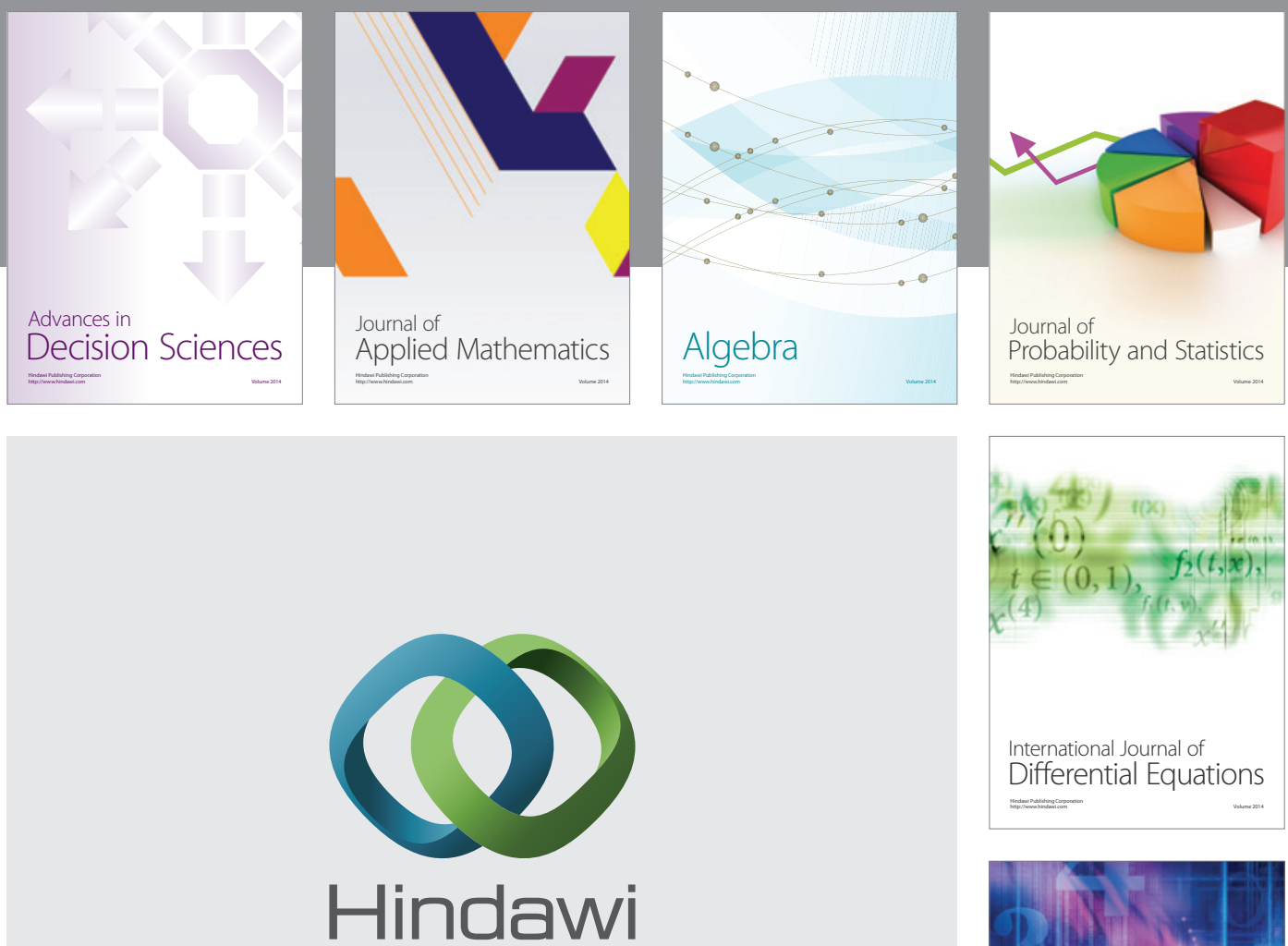

Submit your manuscripts at http://www.hindawi.com
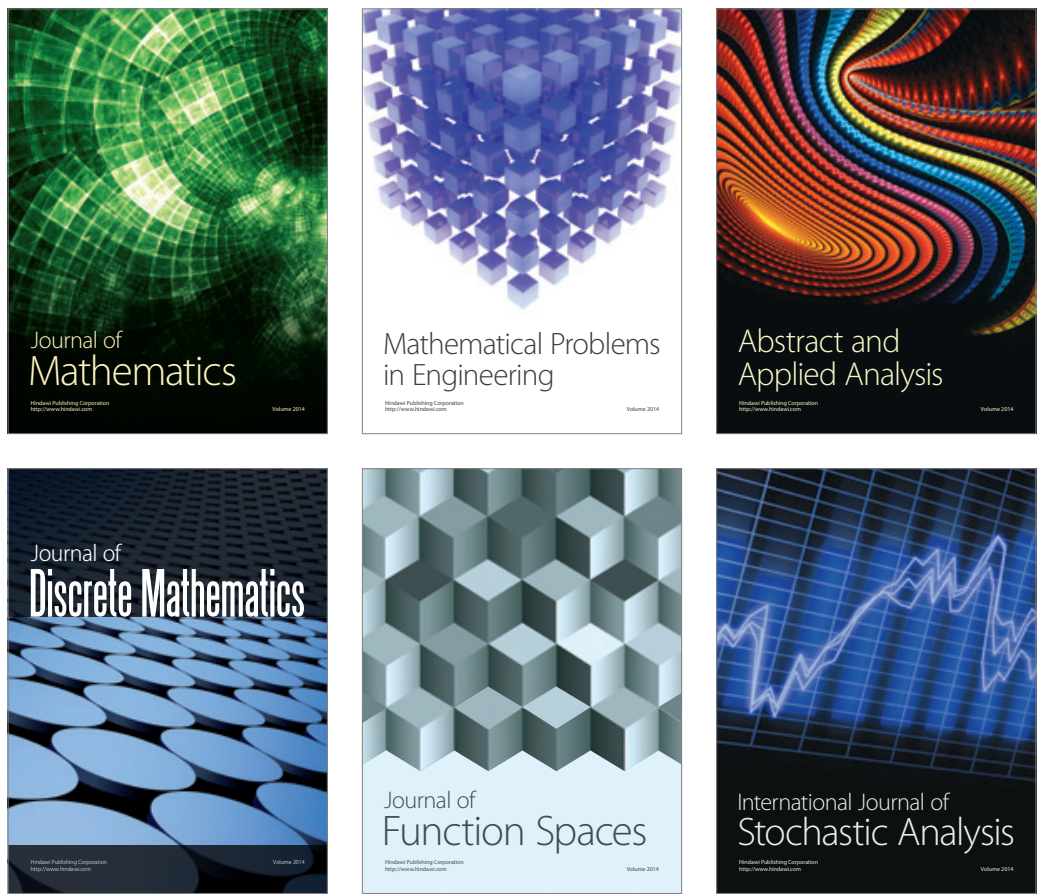

Journal of

Function Spaces

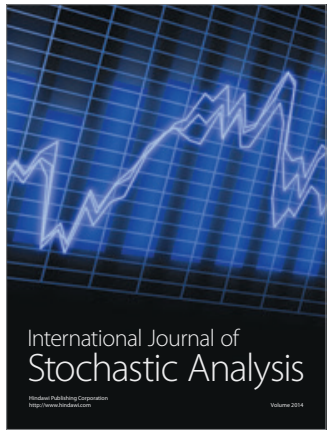

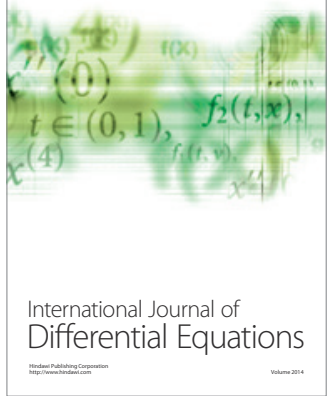
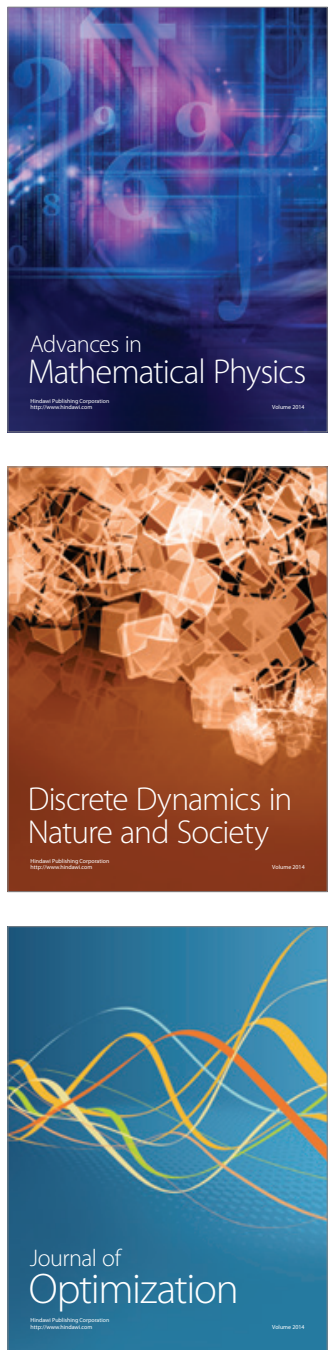